\title{
Caracterización Química y Actividad Antibacteriana in vitro de un Extracto Etanólico de Propóleo Peruano Frente a Streptococcus mutans
}

\author{
Chemical Characterization and in vitro Antibacterial Activity of a \\ Peruvian Propolis Ethanolic Extract Against Streptococcus mutans
}

Jackeline Luciana Checalla Collatupa ${ }^{1} \&$ Marco Antonio Sánchez-Tito $^{2}$

CHECALLA, C. J. L. \& SÁNCHEZ-TITO, M. A. Caracterización química y actividad antibacteriana in vitro de un extracto etanólico de propóleo peruano frente a Streptococcus mutans. Int. J. Odontostomat., 15(1):145-151, 2021.

RESUMEN: El objetivo del estudio fue caracterizar químicamente un extracto etanólico de propóleo peruano y evaluar su actividad antibacteriana frente a Streptococcus mutans. (S. mutans). Se obtuvo el extracto etanólico de propóleo (EEP) por maceración en alcohol al $70 \%$ durante 15 días, el EEP fue sometido a cromatografía en capa fina para identificar sus componentes químicos. EI EEP fue diluido con agua destilada para obtener concentraciones de $75 \%, 50 \%$ y $25 \%$. La actividad antibacteriana se realizó mediante la prueba de difusión en disco sobre medio Brain Heart infusion agar(BHA) inoculado con S. mutans ATCC ${ }^{\circ} 25175^{\mathrm{TM}}$, se empleó clorhexidina $(\mathrm{CHX})$ al $0,12 \%$ como control positivo. Las placas fueron incubadas por 48 horas a $37^{\circ} \mathrm{C}$ en condiciones de microaerofilia. Posteriormente se realizó la medición de los halos de inhibición con un compás Vernier. Los resultados mostraron que el EEP presenta como principales componentes terpenos, di-terpenos y terpenoidales. Todas las concentraciones del EEP presentaron actividad antibacteriana frente al S. mutans $(25 \%=17,582 \pm 2,578 \mathrm{~mm} ; 50 \%=16,906 \pm 1,892 \mathrm{~mm} ; 75 \%=16,881 \pm$ $2,013 \mathrm{~mm} ; 100 \%=17,201 \pm 1,305 \mathrm{~mm})$; sin embargo, fueron menores al compararlos con CHX al 0,12\% $(24,543 \pm 2,486 \mathrm{~mm})$ $(p<0,05)$. Según la escala de Duraffourd, $S$. mutans fue sensible $(+)$ y muy sensible $(++)$ para todas las concentraciones del EEP, mientras que para $\mathrm{CHX}$ al $0,12 \%$ fue sumamente sensible $(+++)(p<0,05)$. Como conclusión, las diversas concentraciones de EEP peruano presentan actividad antibacteriana significativa considerada como sensible y muy sensible frente a $S$. mutans.

PALABRAS CLAVE: agente antibacteriano, propóleo, Streptococcus mutans.

\section{INTRODUCCIÓN}

La caries dental es una enfermedad multifactorial, que corresponde a una alteración del equilibrio y proporción entre las diversas especies de microorganismos de la flora oral y factores relacionados a la ingesta de alimentos con altos contenidos de azúcar y carbohidratos (Simón-Soro \& Mira, 2015). S. mutans es el principal agente bacteriano en la génesis de la caries dental, se trata de un coco Gram positivo y anaerobio facultativo que frente a dietas altas en carbohidratos produce ácidos orgánicos como resultado del metabolismo del azúcar; estos ácidos son responsables de remover el mineral de la pieza dentaria y posteriormente generar lesiones cavitadas (Fejerskov et al., 2015).
Para la prevención de la caries dental existen diversas estrategias, como una correcta higiene bucal, el uso de productos que contienen xilitol, uso de flúor o consumo de agua fluorada, reducir el consumo de alimentos azucarados o con carbohidratos y también el uso de colutorios antibacterianos como la clorhexidina (Nuñez et al., 2010; Bonetti \& Clarkson, 2016; Janket et al., 2019). Frente a la búsqueda de nuevas propuestas de carácter preventivo o de tratamiento, diversas investigaciones han propuesto el uso de agentes naturales con el objetivo de comprobar su efectividad frente al $S$. mutans u otras bacterias precursoras de la caries (Dona \& Rueda, 2015; Airen et al., 2018).

\footnotetext{
${ }^{1}$ Cirujano Dentista, Práctica privada,

${ }^{2}$ Magister en Investigación Científica e Innovación, Facultad de Ciencias de la Salud, Universidad Privada de Tacna. Doctorado (c) en Estomatología, Perú.
}

Received: 2020-06-15 Accepted: 2020-10-31 
El Perú es un país biodiverso donde existe una gran variedad de recursos naturales, dentro de ellos se destaca el propóleo, que es una sustancia resinosa procesada por las abejas "Apis Mellífera", obtenida de los brotes de plantas, flores, arbustos y que es empleada para la protección de las colmenas (Peña, 2008; Viloria et al., 2012). El propóleo posee ciertas características como ser heterogéneo, espeso, de color amarillo, verde oscuro, pardo, hasta negro; aromático, de sabor amargo y de una consistencia pegajosa cuando recibe temperaturas de más de $15^{\circ} \mathrm{C}$ y líquida a temperaturas entre 60 a 70 ${ }^{\circ} \mathrm{C}$ (Chaillou et al., 2004; Huang et al., 2014). Los propóleos están constituidos principalmente por resinas $50 \%$, cera $30 \%$ polen $5 \%$ aceites esenciales $10 \%$ y otros residuos orgánicos (Huang et al.; Delgado et al., 2015). Las propiedades del propóleo corresponden a sus componentes químicos, en tal sentido diversas investigaciones han reportado más de 300 constituyentes en diversas muestras, los principales componentes son los flavonoides, flavonas, terpenoides, pinocembrin, chalcona, ácido pcumárico, entre otros (Sorkun et al., 2001; Said et al., 2006; Celemli et al., 2013; de Mendonça et al., 2015; Freires et al., 2016).

El propóleo ha sido ampliamente empleado en el área de la salud, como anestésico local, bactericida, bacteriostático, tratamiento de enfermedades respiratorias y de acción dermatológica (Freires et al.). En el campo de la odontología el propóleo también ha sido empleado como bacteriostático, antimicrobiano, antifúngico, anestésico y cicatrizante de los tejidos orales (Airen et al.). Los estudios realizados en Perú para conocer el efecto del propóleo frente a $S$. mutans han empleado el extracto etanólico del propóleo puro o en diluciones (Eguizábal \& Moroni, 2007; Mayta-Tovalino \& Sacsaquispe-Contreras, 2010; Mayta-Tovalino et al., 2012); sin embargo, no se ha realizado la caracterización química de sus componentes, lo cual resultaría importante para poder identificar las propiedades inherentes a esta sustancia, considerando que la diversidad en flora puede afectar la efectividad del producto. Por otro lado, conocer la composición química del propóleo sería de gran utilidad para promover la síntesis de sustancias anticariogénicas y la posterior formulación de productos odontológicos.

El objetivo del estudio fue caracterizar químicamente un extracto etanólico de propóleo peruano a través de cromatografía en capa fina y evaluar su actividad antibacteriana frente a $S$. mutans.

\section{MATERIAL Y MÉTODO}

El presente estudio in vitro, experimental, prospectivo, transversal y analítico, empleó una muestra propóleo peruano obtenido de la Región altoandina de la ciudad de Tacna, Perú. La investigación fue inscrita en la Unidad de Investigación de la Facultad de Ciencias de la Salud de la Universidad Privada de Tacna, y al tratarse de un estudio in vitro donde no existió participación de sujetos ni utilización de tejidos de procedencia humana o animal fue exonerado por el Comité de Ética de la Universidad.

Obtención del extracto etanólico del propóleo. Se recolectaron 200 gr de propóleo del centro de Apicultura Abejas Monteflor Perú - ubicado en la ciudad de Tacna, Perú. En seguida, se separaron algunos componentes como restos pequeños de astillas de madera, hojas u otros, obteniendo una muestra final de $150 \mathrm{gr}$ y fueron almacenados en frascos esteriles bajo refrigeración, hasta su uso.

Para la obtención del EEP se procedió a cortar el propóleo en trozos pequeños para luego ser procesado hasta obtener un polvo fino, posteriormente se colocó $100 \mathrm{gr}$ de propóleo en un frasco de vidrio ámbar estéril y se le agregó $500 \mathrm{~mL}$ de alcohol al $70 \%$, el frasco se colocó en una estufa a $37^{\circ} \mathrm{C}$ por 15 días, el frasco se agitó tres veces por día para asegurar una correcta mezcla de los componentes. El sobrenadante se filtró con papel Wathman $\mathrm{N}^{\circ} 42 \mathrm{y}$ $\mathrm{N}^{\circ} 4$. El EEP fue llevado en un vaso beaker a una estufa a $50{ }^{\circ} \mathrm{C}$ para la eliminación del solvente por 04 días, obteniéndose un producto cristalino ámbar que fue removido del vaso beaker con una espátula metálica, el producto fue homogenizado en un mortero de porcelana, obteniendose $3,5 \mathrm{~g}$ de un polvo fino que fue reactivado con $25 \mathrm{~mL}$ de alcohol al 70 $\%$, obteniendo el EEP puro. Finalmente se colocó $7,5 \mathrm{~mL} ; 5 \mathrm{~mL}$ y $2,5 \mathrm{~mL}$ de agua destilada en distintos frascos ambar donde se les añadió $2,5 \mathrm{~mL} ; 5 \mathrm{~mL}$ y $7,5 \mathrm{~mL}$ del EEP puro, para obtener las concentraciones de $25 \%, 50 \%$ y $75 \%$.

Caracterización química del EEP. La determinación de metabolitos secundarios se realizó bajo la metodología de cromatografía en capa fina (CCF), empleando como fase movil: el acetato de metanol agua (4:0,5: 0,40$)$ y como revelador: vainilla (1\%), ácido sulfúrico $(5 \%)$ bajo condiciones de temperatura a $100{ }^{\circ} \mathrm{C}$. Finalmente, la cromatoplaca se reveló bajo 
una lámpara de luz UV a 365 nm. La CCF se realizó en el laboratorio de ensayo y control de calidad de la Universidad Católica de Santa María.

Cepa Bacteriana. La cepa de S. mutans ATCC $®$ $25175^{\mathrm{TM}}$ fue reactivada en BHA por 24 horas a $37^{\circ} \mathrm{C}$ en en una jarra de anaerobiosis empleando en sistema GaspakTM EZ Campy Container System, para generar un ambiente de microaerofilia. Con un asa bacteriológica se trasfirió 5 colonias de $S$. mutans a un tubo con $5 \mathrm{~mL}$ de Brain Heart infusion Broth $(\mathrm{BHI})$ y fueron incubados por 4 horas. Posteriormente el tubo con $\mathrm{BHI}$ que contenia el crecimiento bacteriano fue ajustado a $1,5 \times 108 \mathrm{UFC} / \mathrm{mL}$, lo que corresponde a un nivel de turbidez de 0,5 de la escala estandar de Mc Farland.

Prueba de sensibilidad antibacteriana. Para evaluar la actividad antibacteriana de las diferentes concentraciones del EEP, se empleó el método de difusión de disco en agar. Se emplearon 12 placas con BHA que fueron sembradas con un hisopo estéril embedido con el inóluco de $S$. mutans contenido en el tubo con BHI. 08 placas fueron utilizadas para colocar 04 discos discos de papel Wathman $\mathrm{N}^{\circ} 3$ estériles de $5 \mathrm{~mm}$ diámetro cargados con $10 \mu \mathrm{L}$ de las concentraciones del EEP. En las cuatro placas restantes se colocaron discos em-

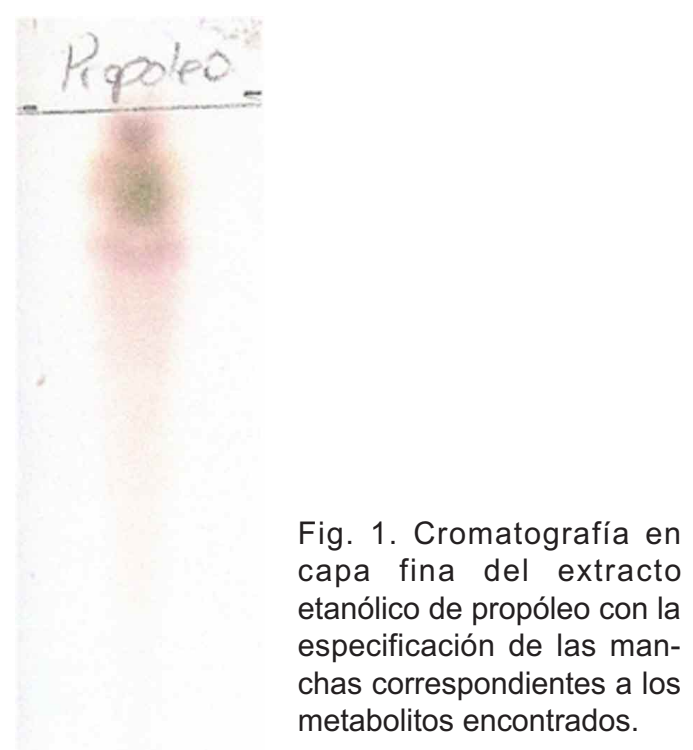

bebidos con $\mathrm{CHX}$ al $0,12 \%$ y agua destilada como control positivo y negativo respectivamente. Las placas fueron colocadas en una jarra de anaerobiosis con un sobre del sistema Gaspak ${ }^{\text {TM }}$ EZ Campy Container System para generar un ambiente de microaerofilia y fueron incubadas por 48 horas a $37^{\circ} \mathrm{C}$. La medición de los halos de inhibición se realizó empleando un compás Vernier digital y los datos fueron anotados en una ficha de registro.

Análisis de los datos. El análisis estadístico fue desarrollado con el programa SPSS para Mac OS en su versión 23.0 (SPSS Inc., Chigago IL, USA). El conjunto de valores medidos con el compás Vernier fueron analizados con la prueba de Shapiro-Wilk para verificar el comportamiento de la distribución de los datos. Se seleccionó la prueba de Kruskall-Wallis para identificar la existencia de diferencias entre los grupos. Adicionalmente, los datos fueron interpretados bajo los criterios de Duraffourd et al. (1986), para ello se empleó la prueba de Xi2 de Pearson. El nivel de significancia adoptado para todas las pruebas fue del $5 \%$.

\section{RESULTADOS}

El análisis por cromatografía en capa fina realizado al EEP puro identificó la presencia de terpenos, di-terpenos y terpenoidalescomo metabolitos secundarios. El revelado de la cromatoplaca donde se observan las manchas correspondientes a los metabolitos hallados se muestra en la Figura 1.

En la Tabla I se muestran los valores promedios de halos de inhibición de los grupos experimentales frente a la cepa de S. mutans. El EEP al $25 \%$ presentó una mayor actividad antibacteriana con halos de inhibición de $17,582 \mathrm{~mm}( \pm 2,578)$ en comparación a las otras concentraciones de EEP al $50 \%, 75 \%$ y $100 \%$, aunque no representó un diferencia estadísticamente significativa $(p>0,05)$ entre ellos. Por otro lado, la $\mathrm{CHX}$ al 0,12 $\%$ demostró ser más efectiva que las concentraciones de EEP $(p<0,05)$, generando halos de inhibición de $24,543 \mathrm{~mm}( \pm 2,486)$. Las comparaciones entre las distintas concentraciones de EEP y $\mathrm{CHX}$ al $0,12 \%$ se muestran en la Figura 2.

En la Figura 3 se puede observar el detalle de los halos de inhibición generados por las distintas concentraciones del EEP en placa Petri inoculada con $S$. mutans; se observa que las cuatro concentraciones generaron halos de inhibición bien definidos. 
Tabla I. Actividad antibacteriana de las concentraciones de EEP sobre S. mutans (valores en mm).

\begin{tabular}{lccccc}
\hline Grupos & $\mathrm{n}$ & Media* $^{*}$ & D.E. & Valor mínimo & Valor máximo \\
\hline EEP $25 \%$ & 08 & $17,582^{\mathrm{a}}$ & 2,578 & 11,62 & 19,76 \\
EEP $50 \%$ & 08 & $16,906^{\mathrm{a}}$ & 1,892 & 14,34 & 20,41 \\
EEP $75 \%$ & 08 & $16,881^{\mathrm{a}}$ & 2,013 & 14,49 & 20,42 \\
EEP $100 \%$ & 08 & $17,201^{\mathrm{a}}$ & 1,305 & 15,48 & 19,55 \\
CHX 0,12\% & 08 & $24,543^{\mathrm{b}}$ & 2,486 & 21,25 & 28,18
\end{tabular}

* Valores con letras diferentes denotan diferencias significativas entre ellos. Prueba de Kruskal-Wallis $(p<0,05)$. D.E.: desviación estándar.

Se empleó la escala de Duraffourd y Lampraz para identificar el grado de sensibilidad de las distintas concentraciones del EEP frente a S. mutans. Todas las contentraciones del EEP fueron sensibles $(+)$ y muy sensibles (++), mientras que la $\mathrm{CHX}$ al $0,12 \%$ fue sumamente sensible (+++); la diferencia en la comparación entre los grupos experimentales y el grado de sensibilidad demostró ser estadísticamente significativa $(\mathrm{p}$ $<0,05)$ (Tabla II).

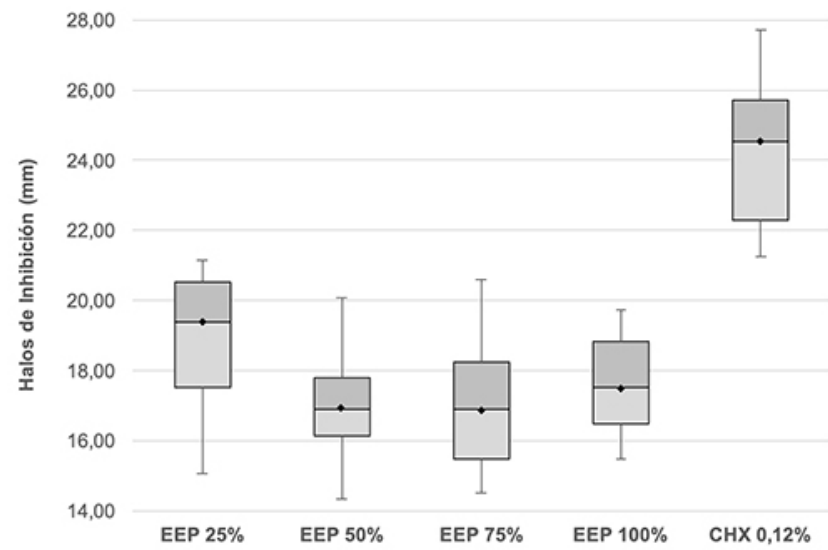

Fig. 2. BoxPlot para la comparación de los halos de inhibición generados por las diferentes concentraciones del EEP y clorhexidina al $0,12 \%$ sobre $S$. mutans.

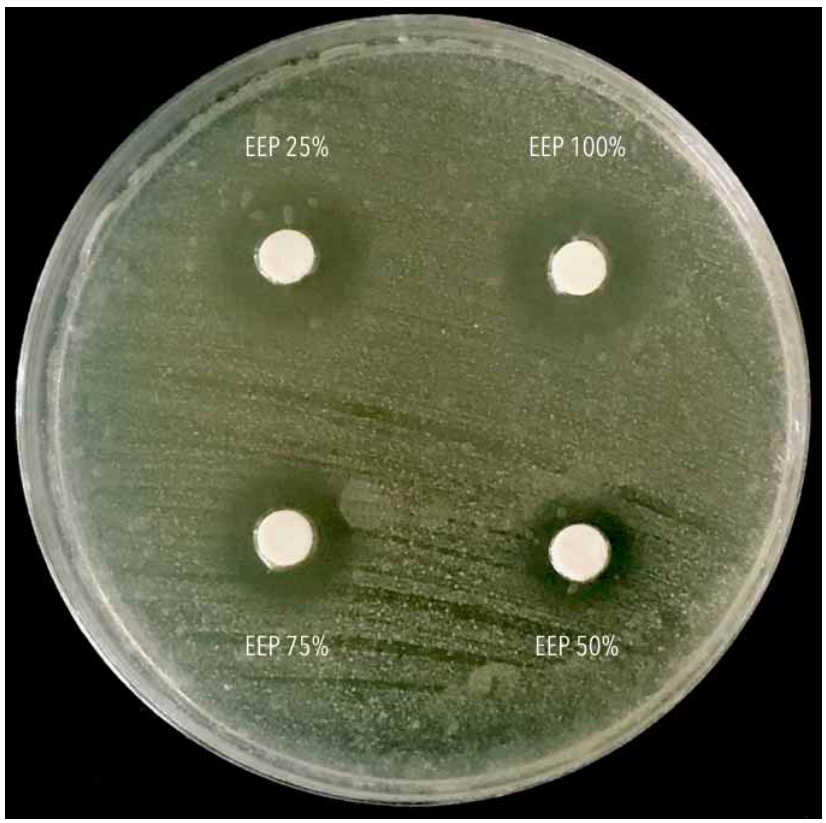

Fig. 3. Halos de inhibición generados por las concentraciones del EEP sobre placa Petri inoculada con S. mutans.

Tabla II. Grado de sensibilidad del S. mutans a las diferentes concentraciones del EEP según la Escala de Sensibilidad de Duraffourd et al.

\begin{tabular}{|c|c|c|c|c|c|}
\hline \multicolumn{6}{|c|}{ Escala de Sensibilidad de Duraffourd et al. } \\
\hline Grupos & $\begin{array}{c}\text { Nula } \\
(-)\end{array}$ & $\begin{array}{c}\text { Sensible } \\
(+)\end{array}$ & $\begin{array}{l}\text { Muy sensible } \\
\qquad(++)\end{array}$ & $\begin{array}{c}\text { Sumamente } \\
\text { sensible }(+++)\end{array}$ & Valor $p^{*}$ \\
\hline EEP $25 \%$ & $0(0,0 \%)$ & $1(12,5 \%)$ & $7(87,5 \%)$ & $0(0,0 \%)$ & \\
\hline EEP $50 \%$ & $0(0,0 \%)$ & $2(25,5 \%)$ & $5(62,5 \%)$ & $1(12,5 \%)$ & \\
\hline EEP $75 \%$ & $0(0,0 \%)$ & $2(25,5 \%)$ & $5(62,5 \%)$ & $1(12,5 \%)$ & 0,000 \\
\hline EEP $100 \%$ & $0(0,0 \%)$ & $0(0,0 \%)$ & $8(100,0 \%)$ & $0(0,0 \%)$ & \\
\hline $\mathrm{CHX} 0,12 \%$ & $0(0,0 \%)$ & $0(0,0 \%)$ & $0(0,0 \%)$ & $8(100,0 \%)$ & \\
\hline
\end{tabular}

*La comparación por medio de la prueba de $\mathrm{Xi}^{2}$ de Pearson mostró diferencias significativas entre los grupos. 


\section{DISCUSIÓN}

La literatura ha reportado extensamente la actividad antibacteriana de productos naturales contra diversos agentes patógenos, dentro de ellos el propóleo. En este estudio, el EEP fue caracterizado químicamente a través de cromatografía en capa fina, identificandose la presencia de terpenos, di terpenos y terpenoidales. Una gran variedad en la composición química de propóleos de diversas regiones puede encontrarse en la literatura (Huang et al.; Sorkun et al.; Said et al.; Celemli et al.; de Mendonça et al.; Freires et al.), esto puede estar relacionado a las diferencias inherentes de la región de donde se colecta la muestra, condiciones clímaticas, la flora y el método de análisis. Sorkun et al. detectaron como mayor componente ácidos derivados de la quetona en muestras de propóleo de Turquía. Barrientos et al. (2013) identificaron los componentes de diversas muestras de propóleo chileno, dentro de los cuales pinocembrin, galangina, quercetina, apigenina, y ácido caféico quercetina, fueron los más frecuentes. De Mendoça et al. analizaron el perfil fitoquímico del propóleo brasilero identificando como principales componentes a los taninos, flobafenos, catequinas, chalconas y flavononas.

La actividad antibacteriana del EEP ha sido reportada frente al diversos microorganismos, incluidos aquellos de importancia para la odontología. En ese sentido, la actividad antibacteriana in vitro del EPP frente al S. mutans fue investigada. Los resultados mostraron que el EPP es efectivo para inhibir el crecimiento de $S$. mutans cuando se evaluó a través de la prueba de difusión de disco en agar. Se emplearon $10 \mu \mathrm{L}$ del EEP que contenían $8,35 \mu \mathrm{g}$ de propóleo. Los halos de inhibición fueron similares para las cuatro concentraciones estudiadas, sin embargo la $\mathrm{CHX}$ al $0,12 \%$ fue más efectiva para inhibir el crecimiento del S. mutans. Kim et al. (2011) determinaron que concentraciones mayores de $35 \mu \mathrm{g} / \mathrm{mL}$ de EEP presentaron actividad antibacterana frente a $S$. mutans y S. sobrinus. Barrientos et al. evaluaron la actividad antibacteriana y concentración mínima inhibitoria por método de microdilución del EEP chileno frente a $S$. mutans, demostrando un amplio espectro de acción desde 0,9 a $8,22 \mu \mathrm{g} / \mathrm{mL}$. Eguizábal \& Moroni evaluaron la actividad antibacteriana del EEP al $0,8, \% 20 \%, 30 \%$ frente a S. mutans, sus resultados mostraron una tendencia de inhibición inversamente proporcial a su concentración, concluyendo que el EEP proveniente de la región de Oxapampa es efectivo para la inhibición del crecimiento del S. mutans, siendo que el EEP al $0,8 \%$ presentó un promedio de halos de inhi- bición de 13,56 $\pm 3,04 \mathrm{~mm}$, estos resultados fueron mayores incluso que los registrados para la $\mathrm{CHX}$ al 0,12 $\%(11,72 \pm 1,50 \mathrm{~mm})$. Mayta-Tovalino \& SacsaquispeContreras evaluaron el EEP en concentraciones del 10 y $30 \%$, los halos de inhibición fueron similares para ambas concentraciones (EEP $10 \%$ media 11,47 $\pm 0,57$ $\mathrm{mm}$ y EEP $30 \%$ media $11,57 \pm 0,97 \mathrm{~mm}$ ), ambas concentraciones mostraron resultados similares a los registrados para la $\mathrm{CHX}$ al $0,12 \%(11,77 \mathrm{~mm})$. Airen et al. demostraron que el EEP procedente de la India a concentraciones del 5 y $20 \%$ fueron efectivos para inhibir el crecimiento de $S$. mutans empleando la técnica de difusión en placa.

Duarte et al. (2006) indican que las propiedades biológicas del propóleo, como la disminución de la tolererancia de los microorganismos a pH bajos y la limitación de la formación de biofilm sobre el sustrato dentario, son debidas a sus componentes como el ácido oléico, linoléico y esteárico presentes, que son capaces de inhibir la actividad de la enzima glucosiltransferasa (GTF) que es la precursora de la adhesión del $S$. mutans a la superficie dentaria. Park et al. (1998) identificaron que el EEP de diversas zonas de Brasil presentan la capacidad de inhibir la GTF de estreptococos del grupo mutans y que el principal componente asociado a esta actividad fue pinocembrin. Resultados similares han sido reportados por Koo et al. (2002) indicando que las flavonas como la apigenina y los flavonoles tienen la capacidad de inhibir la actividad de la GTF.

El EEP es una mezcla de componentes químicos y es dificil atribuir las propiedades biológicas de uno o un grupo de ellos. Existen diversas metodologías que permiten fraccionar y extraer por medio de solventes orgánicos los componentes de los productos naturales y de esta forma aislar e identificar sus propiedades. En nuestro estudio, los metabolitos secundarios encontrados en el EEP fueron terpenos, di-terpenos y terpenoidales, lo que podría explicar el efecto inhibitorio sobre el crecimiento del $S$. mutans.Sin embargo, llama la atención la ausencia de flavonoides, ya que otros estudios reportaron la presencia de estos como los principales componentes de las muestras de EEP, aunque también se sabe que el lugar de recolección, la temporada en la que se realizó, la flora de la zona pueden modificar la composición de las muestras. Además, estos resultados representan el análisis del EEP puro y bajo una metodología cualitativa y deben realizarse otros ensayos más específicos para la identificación de los compuestos como las partición y fraccionamiento del EEP y técnicas cromatográficas como la de gases 
acoplada a espectrometría de masas y cromatografía líquida de alta eficacia para poder aislar y cuantificar sus componentes químicos y poder promover el desarrollo de modelos de nuevos productos para el control de la caries dental.

\section{CONCLUSIONES}

Como conclusión, el análisis de cromatografía en capa fina del EEP peruano mostró una mezcla de metabolitos secundarios como terpenos, di-terpenos y terpenoidales. Además, el EEP exhibió actividad antibacteriana en todas las concentraciones estudiadas siendo sensible y muy sensible frente a $S$. mutans, aunque los resultados fueron menores que los registrados para la $\mathrm{CHX}$ al $0,12 \%$.El propóleo ha sido empleado para el tratamiento de muchas enfermedades en la medicina tradicional y considerando su actividad frente a S. mutans, puede ser considerado para mayores estudios con el objetivo de desarrollar nuevas estratégias para el control de la caries y además comprender completamente su bioactividad ya que el efecto del EEP sobre $S$. mutans puede ser debido a un sinergismo entre sus componentes.

CHECALLA, C. J. L. \& SÁNCHEZ-TITO, M. A. Chemical characterization and in vitro antibacterial activity of a Peruvian propolis ethanolic extract against Streptococcus mutans. Int. J. Odontostomat., 15(1):145-151, 2021.

ABSTRACT: The objective of the study was to chemically characterize an ethanolic extract of Peruvian propolis and evaluate its antibacterial activity against Streptococcus mutans. (S. mutans). The ethanolic extract of propolis (EEP) was obtained by maceration in $70 \%$ alcohol for 15 days, the EEP was subjected to thin layer chromatography to identify its chemical components. The EEP was diluted with distilled water to obtain concentrations of 75 $\%, 50 \%$ and $25 \%$. The antibacterial activity was performed by the disk diffusion test on Brain Heart infusion agar (BHA) medium inoculated with S. mutans ATCC $\otimes 25175$ TM, $0.12 \%$ chlorhexidine $(\mathrm{CHX})$ was used as a positive control. Plates were incubated for 48 hours at $37^{\circ} \mathrm{C}$ under microaerophilic conditions. Subsequently, the inhibition halos were measured with a Vernier compass. The results showed that the EEP presents as main components terpenes, di-terpenes and terpenoidals. All concentrations of the EEP showed antibacterial activity against $S$. mutans $(25 \%=17,582 \pm 2,578$ $\mathrm{mm} ; 50 \%=16,906 \pm 1,892 \mathrm{~mm} ; 75 \%=16,881 \pm 2,013 \mathrm{~mm}$; $100 \%=17,201 \pm 1,305 \mathrm{~mm})$; however, they were lower when compared with $0.12 \% \mathrm{CHX}(24,543 \pm 2,486 \mathrm{~mm})(p<0.05)$. According to the Duraffourd' scale, S. mutans was sensitive
$(+)$ and very sensitive $(++)$ for all EEP concentrations, while for $0.12 \% \mathrm{CHX}$ it was highly sensitive $(+++)(p<0.05)$. In conclusion, all concentrations of Peruvian EEP have significant antibacterial activity considered as sensitive and very sensitive against $S$. mutans.

KEY WORDS: antibacterial agent, propolis, Streptococcus mutans.

\section{REFERENCIAS BIBLIOGRÁFICAS}

Airen, B.; Sarkar, P. A.; Tomar, U. \& Bishen, K. A. Antibacterial effect of propolis derived from tribal region on Streptococcus mutans and Lactobacillus acidophilus: An in vitro study. J. Indian Soc. Pedod. Prev. Dent., 36(1):48-52, 2018.

Barrientos, L.; Herrera, C. L.; Montenegro, G.; Ortega, X.; Veloz, J.; Alvear, M.; Cuevas, A.; Saavedra, N. \& Salazar, L. A. Chemical and botanical characterization of Chilean propolis and biological activity on cariogenic bacteria Streptococcus mutans and Streptococcus sobrinus. Braz. J. Microbiol., 44(2):577-85, 2013.

Bonetti, D. \& Clarkson, J. E. Fluoride varnish for caries prevention: Efficacy and implementation. Caries Res., 50 Suppl. 1:45-9, 2016.

Celemli, O. G.; Hatjina, F.; Charistos, L.; Schiesser, A. \& Özkirim, A. More insight into the chemical composition of Greek propolis; differences and similarities with Turkish propolis. Z. Naturforsch. C. J. Biosci., 68(11-12):429-38, 2013.

Chaillou, L. L.; Herrera, H. A. \& Maidana, J. F. Estudio del propóleos de Santiago del Estero, Argentina. Ciênc. Tecnol. Aliment., 24(1):11-5, 2004.

de Mendonça, I. C.; Porto, I. C.; do Nascimento, T. G.; de Souza, N. S.; Oliveira, J. M.; Arruda, R. E.; Mousinho, K. C.; dos Santos, A. F.; Basílio-Júnior, I. D.; Parolia, A.; et al. Brazilian red propolis: phytochemical screening, antioxidant activity and effect against cancer cells. BMC Complement. Altern. Med., 15:357, 2015.

Delgado, A. M. L.; Andrade, O. J. A. \& Ramírez, B. C. A. Caracterización fisicoquímica de propóleos colectados en el bosque La Primavera Zapopan, Jalisco. Rev. Mex. Cienc. For., 6(28):74-87, 2015.

Duarte, S.; Rosalen, P. L.; Hayacibara, M. F.; Cury, J. A.; Bowen, W. H.; Marquis, R. E.; Rehder, V. L.; Sartoratto, A.; Ikegaki, M. \& $\mathrm{Koo}, \mathrm{H}$. The influence of a novel propolis on mutans streptococci biofilms and caries development in rats. Arch. Oral Biol., 51(1):1522,2006

Duraffourd, C.; Hervicourt, L. D. \& Lapraz, J. C. Cuadernos de Fitoterapia Clínica. Barcelona, Masson, 1986.

Eguizábal, A. M. \& Moroni, N. H. Actividad antibacteriana in vitro del extracto etanólico de propóleo peruano sobre Streptococcus mutans y Lactobacillus casei. Odontol. Sanmarquina, 10(2):18-20, 2007.

Fejerskov, O.; Nyvad, B. \& Kidd, E. Dental Caries, the Disease and Its Clinical Management. 3rd ed. Oxford, Wiley Blackwell, 2015.

Freires, I. A.; Queiroz, V. C. P. P.; Furletti, V. F.; Ikegaki, M.; de Alencar, S. M.; Duarte, M. C. T. \& Rosalen, P. L. Chemical composition and antifungal potential of Brazilian propolis against Candida spp. J. Mycol. Med., 26(2):122-32, 2016

Huang, S.; Zhang, C. P.; Wang, K.; Li, G. Q. \& Hu, F. L. Recent advances in the chemical composition of propolis. Molecules, 19(12):19610-32, 2014

Janket, S. J.; Benwait, J.; Isaac, P.; Ackerson, L. K. \& Meurman, J. H. Oral and systemic effects of xylitol consumption. Caries Res., 53(5):491-501, 2019. 
Kim, M. J.; Kim, C. S.; Kim, B. H.; Ro, S. B.; Lim, Y. K.; Park, S. N.; Cho, E.; Ko, J. H.; Kwon, S. S.; Ko, Y. M.; et al. Antimicrobial effect of Korean propolis against the mutans streptococci isolated from Korean. J. Microbiol., 49(1):161-4, 2011.

Koo, H.; Rosalen, P. L.; Cury, J. A.; Park, Y. K. \& Bowen, W. H. Effects of compounds found in propolis on Streptococcus mutans growth and on glucosyltransferase Activity. Antimicrob. Agents Chemother., 46(5):1302-9, 2002.

Mayta-Tovalino, F.; Sacsaquispe, C. S.; Ceccarelli, C. J. F. \& Alania, M. J. Propóleo Peruano: Una nueva alternativa terapéutica antimicrobiana en Estomatología. Rev. Estomatol. Herediana, 22(1):50-8, 2012.

Mayta-Tovalino. F. \& Sacsaquispe-Contreras S. J. Evaluación in vitro del efecto antibacteriano del extracto etanólico de propóleo de Oxapampa - Perú sobre cultivos de Streptococcus mutans (ATCC 25175) y Staphylococcus aureus (ATCC 25923). Rev. Estomatol. Herediana, 20(1):19-24, 2010.

Nuñez, D. P. \& García Bacallao, L. Bioquímica de la caries dental. Rev. Habanera Cienc. Méd., 9(2):156-66, 2010.

Park, Y. K.; Koo, M. H.; Abreu, A. S.; Ikegaki, M.; Cury, J. A. \& Rosalen, P. L. Antimicrobial activity of propolis on oral microorganisms. Curr. Microbiol., 36(1):24-28, 1998.

Peña, R. C. Estandarización en propóleos: antecedentes químicos y biológicos. Cienc. Investig. Agrar., 35(1):17-26, 2008.

Said, S. A.; Khan, S. A.; Ahmad, I. \& Ali, H. S. Chemical composition of Egyptian and UAE propolis. Pak. J. Pharm. Sci., 19(1):58-61, 2006.

Simón-Soro, A. \& Mira, A. Solving the etiology of dental caries. Trends Microbiol., 23(2):76-82, 2015.

Sorkun, K.; Süer, B. \& Salih, B. Determination of chemical composition of Turkish propolis. Z. Naturforsh. C. J. Biosci., 56(7-8):666-8, 2001.

Viloria, B. J. D.; Gil, G. J. H.; Durango, R. D. L. \& García, P. C. M. Caracterización fisicoquímica del propóleo de la región del bajo Cauca Antioqueño (Antioquia, Colombia). Rev. Bio. Agro., 10(1):77-86, 2012.

\section{Dirección para correspondencia:}

Marco A. Sánchez-Tito

Facultad de Ciencias de la Salud

Universidad Privada de Tacna

Av. Jorge Basadre Grohmann s/n,

Tacna

PERÚ

Email: marcosanchez2183@gmail.com 